\title{
Testing the null hypothesis of the nonexistence of a preseizure state
}

\author{
Ralph G. Andrzejak, ${ }^{1, *}$ Florian Mormann, ${ }^{2,3}$ Thomas Kreuz, ${ }^{1,2}$ Christoph Rieke, ${ }^{2,3}$ Alexander Kraskov, ${ }^{1}$ Christian E. Elger, ${ }^{2}$ \\ and Klaus Lehnertz ${ }^{2}$ \\ ${ }^{1}$ John-von-Neumann Institute for Computing, Forschungszentrum Jülich, 52425 Jülich, Germany \\ ${ }^{2}$ Department of Epileptology, University of Bonn, Sigmund-Freud-Straße 25, 53105 Bonn, Germany \\ ${ }^{3}$ Helmholtz. Institut für Strahlen- und Kernphysik, University of Bonn, Nußallee 14-16, 53115 Bonn, Germany
}

(Received 16 June 2002; published 7 January 2003)

\begin{abstract}
A rapidly growing number of studies deals with the prediction of epileptic seizures. For this purpose, various techniques derived from linear and nonlinear time series analysis have been applied to the electroencephalogram of epilepsy patients. In none of these works, however, the performance of the seizure prediction statistics is tested against a null hypothesis, an otherwise ubiquitous concept in science. In consequence, the evaluation of the reported performance values is problematic. Here, we propose the technique of seizure time surrogates based on a Monte Carlo simulation to remedy this deficit.
\end{abstract}

DOI: 10.1103/PhysRevE.67.010901

PACS number(s): 87.19.La, 05.45.Tp, 84.35.+i

Epilepsies are characterized by recurrent and often severe malfunctions of the brain that manifest themselves as epileptic seizures. Most epilepsy patients experience the onset of a seizure as a sudden and unexpected event. Guided by both $a$ priori and a posteriori considerations, however, it has been hypothesized that the transition to the seizure (ictal) state might not be an abrupt phenomenon but rather evolves via a temporally extended preictal state (e.g., Ref. [1]). Provided that such a preictal state detection could be achieved with a sufficient sensitivity and specificity, seizure anticipation and prevention technologies could be envisaged which would be of great benefit for epilepsy patients. In Refs. [2-4], it has been investigated whether information about an impending seizure can be extracted from the electroencephalogram (EEG) using different characterizing measures derived from linear or nonlinear time series analysis. Common to these studies is a two-step procedure: First, a characterizing measure is calculated for a multichannel EEG using a movingwindow technique. In a second step, the resulting spatiotemporal profile of the characterizing measure is analyzed by means of an often highly elaborated evaluation scheme aiming at an extraction of information specific for the preictal state. As distinct and complementary as the different approaches are, in the context of the present study, they will be termed as seizure prediction statistics in their collectivity. Their output in terms of sensitivity and specificity will be denoted as performance.

Let us now consider the following null hypothesis: "The transition from the interictal to the ictal state is an abrupt phenomenon. An intermediate preictal state does not exist." Despite the fact that in this case, no information predictive of impending seizures could be extracted from the EEG, many of the seizure prediction statistics would probably still render nonzero performance values. Moreover, an a priori estimation of these performance values is problematic. Hence, it is impossible to decide whether a given performance value obtained from real data indicates the existence of a preictal state or whether it is consistent with the null hypothesis stated above.

*Electronic address: r.g.andrzejak@fz-juelich.de
A similar problem is known from the application of nonlinear time series analysis techniques to stochastic dynamics. The framework of nonlinear time series analysis comprises of a number of measures that allow the characterization of nonlinear deterministic dynamics [5]. For most of these measures, however, the range of values obtained for nonlinear deterministic dynamics and for linear stochastic dynamics overlap substantially [6]. It is, therefore, impossible to decide whether a given value of a nonlinear measure calculated from some unknown time series reflects a property of an underlying nonlinear deterministic dynamics or whether it is consistent with a linear stochastic model. This ambiguity has been addressed by the method of surrogate data [7]. This method allows the testing of a specified null hypothesis about the dynamics underlying a given time series. For this technique, which can be regarded as a Monte Carlo simulation, an ensemble of surrogate time series is constructed from the original time series in such a way that they have all the properties that are consistent with this null hypothesis in common with the original, but are otherwise random. A discriminating statistics, which has to be sensitive to at least one property that is not consistent with the null hypothesis, is calculated for both the original time series and the surrogates. In case the null hypothesis is the assumption of a linear stochastic process, a measure derived from nonlinear time series analysis can be used as a discriminating statistics. If the result for the original deviates from the distribution of the values obtained from the surrogates, the null hypothesis can be rejected at a level of significance determined by the number of surrogates used. In the beginning, the method of surrogates was mostly used to test the null hypothesis of a linear stochastic model and was regarded exclusively as a test for nonlinearity. Later, it has been understood to be a more general, and therefore, also a more powerful concept. In Ref. [8], nonlinearity was even explicitly included in a null hypothesis. Furthermore, surrogate algorithms have been developed that allow the testing of almost arbitrary null hypotheses [9]. Problems associated with false positive rejections of null hypotheses have been discussed in Ref. [10].

In this paper, we propose a further generalization of the concept of surrogates by constructing seizure time surrogates 


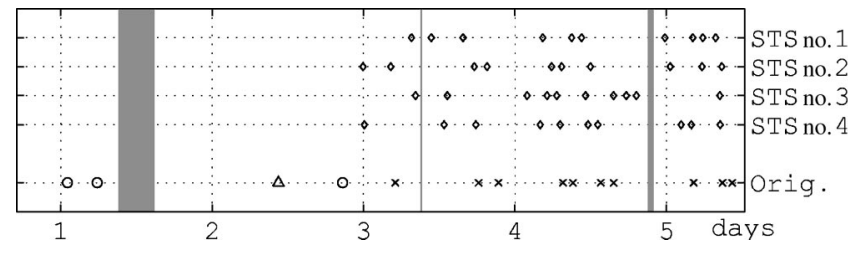

FIG. 1. Temporal distribution of relevant events that occurred during a six-day EEG recording from an epilepsy patient (lower row: $\times$ seizures, $\bigcirc$ subclinical events, $\triangle$ hyperventilation, and gray vertical bars: discontinuities. Four exemplary seizure time surrogates are displayed in the upper rows. Diamonds denote surrogate seizure onset times established by the constrained randomization procedure described in the text.

that allow one to validate the results of seizure prediction statistics. Given a continuous EEG recording, seizure time surrogates can be constructed by replacing the original seizure times with times randomly chosen from the interictal intervals. Specified properties of the original sequence can be imposed as constraints on the surrogate seizure onset times. Subsequently, any given seizure prediction statistics can be carried out for both the original seizure times and the surrogates. Provided that a preictal state exists and the prediction statistics is able to detect it, the statistics' performance should be highest for the original seizure times. A similar approach is used in seismology, where null hypothesis tests are regarded as inevitable to evaluate the performance of earthquake prediction algorithms [11].

To illustrate this technique, we analyzed the spatiotemporal distribution of a nonlinear measure that was calculated from a quasicontinuous EEG recorded over six days during the presurgical work up [12] of an epilepsy patient independently from the design of our retrospective study. Using implanted electrodes, equipped with a total of 48 separate contacts, the EEG was measured directly at the surface of the cortex and within deeper structures of the brain. The EEG data was sampled at $200 \mathrm{~Hz}$ using a 16 bit analog-to-digital converter and filtered within a frequency band of $0.53-100$ $\mathrm{Hz}$.

Figure 1 shows a scheme of events that took place during the recording time and that have to be taken into account for the generation of surrogate seizure onset times. Twice the patient was briefly (13 and $54 \mathrm{~min}$ ) disconnected from the EEG acquisition system. A longer discontinuity (340 min) was necessary to carry out a magnetic resonance imaging scan to determine the exact location of the implanted electrodes. All ten seizures occurred spontaneously within the second half of the recording. The latency of the first seizure can be explained by the remaining effect of antiepileptic drugs that were withdrawn after implantation of the electrodes. During the first three days, only three subclinical events took place, i.e., events during which seizurelike activity can be observed in the EEG while the patient does not show any clinical signs of an ongoing seizure. On the third day, the patient was asked to perform a hyperventilation, a seizure provocation technique that may cause alterations of the EEG. For our study, four intervals of $20 \mathrm{~min}$ starting at the beginning of both the hyperventilation and the three subclinical events, as well as ten intervals of $1 \mathrm{~h}$ starting at the onset of the seizures were excluded from the analysis. The last step was carried out since the ictal and postictal EEG differs substantially from the EEG recorded during the interictal state. Both these exclusions and the aforementioned discontinuities will be referred to as recording gaps. The remaining length of the analyzed EEG amounted to $101.1 \mathrm{~h}$.

In total, 19 seizure time surrogates were generated by replacing original seizure times with times randomly chosen in the interictal intervals (cf. Fig. 1). The following properties of the original seizure times were imposed as constraints on the seizure time surrogates: The total number of seizures $(=10)$, the distribution of intervals between consecutive seizures, and the clustering of the seizures in the second half of the recording. The intervals between consecutive seizures and the interval from the first seizure back to an arbitrarily defined starting point $T_{0}$ at 12 a.m. on the third day are called $D_{1}, \ldots, D_{10}$. For the generation of each of the seizure time surrogates, the following steps were carried out: First, a new starting point was defined as $T_{0}^{*}=T_{0}-\varepsilon(1 \mathrm{~h})$, with $\varepsilon$ being a random number uniformly distributed in $[0,4]$. Starting at $T_{0}^{*}$, surrogate seizure onset times $T_{1}^{*}, \ldots, T_{10}^{*}$ were generated from a random permutation of $D_{1}, \ldots, D_{10}$. The sequence was discarded whenever a recording gap was located within the last hour prior to any of the $T_{1}^{*}, \ldots, T_{10}^{*}$.

As a characterizing measure of the EEG, we used the degree of nonlinear determinism $\xi$. Following Ref. [13], $\xi$ was defined from a combination of the coarse grained flow average $\Lambda$ [14] and iterative amplitude adjusted surrogates [15]. As a direct test for determinism, $\Lambda$ quantifies the alignment of nearby trajectory segments in state space. Here, the use of surrogates is essential to correct an alignment that is caused by autocorrelations rather than by deterministic dynamics. Using a moving-window technique, the EEG was divided into nonoverlapping segments of 20.48 s $(N=4096$ data points). For each of these segments, a set of four surrogates was generated. The dynamics were reconstructed using the method of delays [16] with a fixed embedding dimension $(m=6)$ and varying time delay $\tau$. We defined $\xi$ $\equiv \sum_{\tau=5}^{20}\left(\Lambda_{E E G}-\left\langle\Lambda_{S U R}\right\rangle\right)(\tau)$, with $\Lambda_{E E G}$ denoting the value obtained for the EEG segment, and $\left\langle\Lambda_{S U R}\right\rangle$ denoting the mean value obtained for the surrogates. All parameters were adopted from a previous study in order to avoid any insample overtraining. Only the number of surrogates was reduced from nine to four since the latter value was found to provide a sufficiently reliable estimate of $\left\langle\Lambda_{S U R}\right\rangle$. A $\xi(t)$ profile was obtained for each of the 48 EEG channels for segments $t=1, \ldots, 17764$. In order to disregard short-term fluctuations and rather focus on long-term trends of $\xi(t)$, a moving-average filter of 11 consecutive segments was applied.

In Ref. [13], we have compared mean $\xi$ values obtained from the interictal EEG recorded from within the epileptic focus and from other brain areas of epilepsy patients. A correct localization of the epileptic focus could be derived from increased values of $\xi$ in all investigated cases. Following the basic concept of Ref. [2], we hypothesized that the preictal 


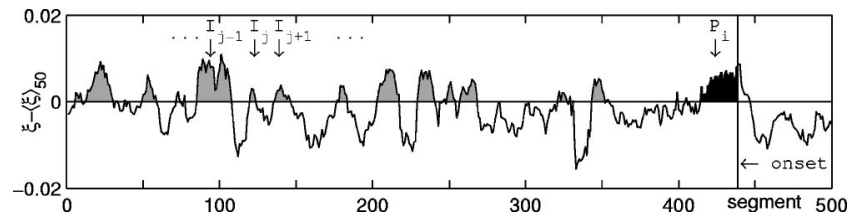

FIG. 2. Parametrization of an exemplary $\xi(t)-\langle\xi\rangle_{50}$ profile. Data from the ictal and postictal state were included for completeness. Gray shaded areas denote interictal peaks $\left(I_{j}\right)$. A preictal peak $\left(P_{i}\right)$ is shown in black.

state would be reflected in an increase of $\xi$ (cf. Ref. [4]), and accordingly designed a simple evaluation for $\xi(t)$ (Fig. 2).

First, a reference level was defined by the median $\langle\xi\rangle_{50}$ of the distribution of all $\xi(t)$ values for each EEG channel. For every interval $B$ between two crossings of $\xi(t)$ and $\langle\xi\rangle_{50}$, we quantify the area $A=\sum_{t \in B}\left[\xi(t)-\langle\xi\rangle_{50}\right]$. The evaluation was restricted to positive areas, which we will refer to as peaks. Let $s$ denote the number of seizures that were directly preceded by a peak instead of a drop of $\xi(t)$ below the reference level. For those seizures, this peak is termed as preictal and its area is denoted by $P_{i}$ for $i=1, \ldots, s$. All other peaks are termed as interictal and their areas are denoted by $I_{j}$ for $j=1, \ldots, k$, with $k$ being the total number of interictal peaks. The $P_{i}$ were only integrated up to the seizure onset times. In order to compare the distributions of $P_{i}$ and $I_{j}$, we calculated $f \equiv\left(\left\langle P_{i}\right\rangle_{50}-\left\langle I_{j}\right\rangle_{50}\right) /\left(\left\langle P_{i}\right\rangle_{50}+\left\langle I_{j}\right\rangle_{50}\right)$ from the medians of the two distributions. Finally, we defined $F$ $\equiv\langle f\rangle$ as the average over all channels. By construction, $f$ and $F$ are restricted to $[-1,1]$ and should tend to zero if the distributions of preictal and interictal peaks match.

Figure 3 shows the distribution function of $I_{j}$ along with corresponding values of $P_{i}$ determined for the original seizure onset times for one exemplary EEG channel. Among the $s=7$ preictal peaks, five peaks were found whose area exceeded the median area of the interictal peaks. For this channel, we obtained $f=0.94$. After averaging the results over all channels, we obtained $F=0.81$ for the original seizure times. At first glance, this value appears quite promising in the sense that it might indicate that the preictal peaks were more pronounced than the interictal peaks, confirming that the preictal state is indeed reflected in an increase of $\xi$.

This interpretation, however, does not necessarily hold: Suppose we selected peaks randomly from a sequence like the one depicted in Fig. 1. If the probability to be selected

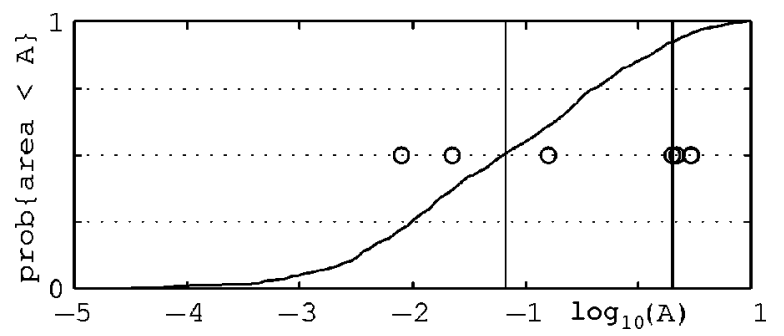

FIG. 3. Distribution function prob $\{$ are $a<A\}$ for interictal peaks $I_{j}$ (line) along with values $P_{1}, \ldots, P_{7}$ (circles) obtained for the original seizure times for one exemplary channel. The two vertical lines indicate the medians $\left\langle I_{j}\right\rangle_{50}$ and $\left\langle P_{i}\right\rangle_{50}$, respectively.

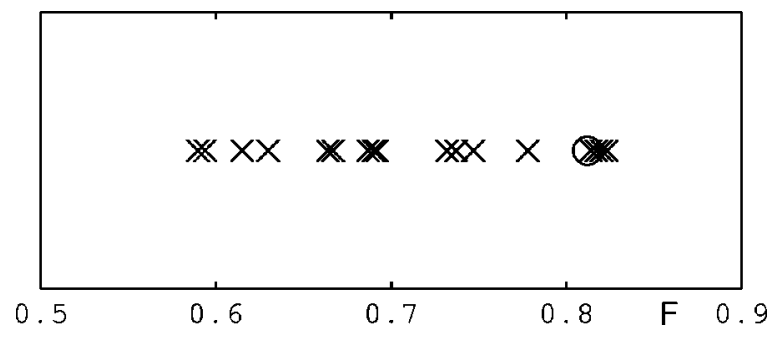

FIG. 4. $F$ values for the original seizure times $(\bigcirc)$ and the distribution of 19 seizure time surrogates $(X)$.

were the same for all peaks, scores with areas below and above the median of the distribution of areas of all peaks would be equiprobable. If we, in contrast, drew samples from such a sequence by randomly selecting points in time, we would be more likely to draw long peaks than to draw short peaks. Consequently, the area of our samples would tend to exceed the aforementioned median. Taking this into account, a value of $F>0$ would be expected even under the assumption of our null hypothesis. Hence, interpreting the significance of the observed value of $F$ appears quite difficult. One could consider a normalization or correction of $F$ based on the distribution function of $I_{j}$ but this might not be sufficient to eliminate any bias caused by further, unforeseen problems and pitfalls.

A more straightforward answer can be obtained from the application of seizure time surrogates. From Fig. 4, it becomes evident that the $F$ value obtained for the original seizure times was within the distribution obtained for the seizure time surrogates. On the level of single EEG channels, i.e., based on $f$ values, the null hypothesis could be rejected for four of the 48 channels. However, if a test with a nominal size of $\alpha=0.05$ is repeated 48 times, there is a $9 \%$ chance to obtain up to four rejections. Hence, we could not reject the null hypothesis of the nonexistence of a preictal state by means of the applied seizure prediction statistics.

The fact that the null hypothesis could not be rejected does by no means prove its correctness. Rather, there exist numerous alternative explanations for this result. For several reasons, the applied seizure prediction statistics might simply lack any discriminative power for the hypothesis test: Even though $\xi$ did allow a characterization of the spatial distribution of the interictal epileptic dynamics [13], it may still be incapable to detect any feature of the EEG specific for the preictal state. An explanation for such a finding would be that the interictal epileptic dynamics and the seizuregenerating process are two distinct dynamical phenomena, each imposing different features on the EEG. On the other hand, even if $\xi$ were capable to detect the preictal state, the relevant information could be missed by our rather simple evaluation of $\xi(t)$. Furthermore, our study was based on the EEG recording of only one epilepsy patient. It would be highly speculative to draw any conclusions about the multifaceted disease epilepsy from such a limited sample. It is far beyond the scope of the present study to prove or disprove the existence of a preictal state. Rather, the aim was to propose a simple technique that allows one to validate the performance of seizure prediction statistics. In some cases, e.g., 
if only a collection of very short recordings each containing one seizure is available, a randomization of seizure onset times might not be possible. In these cases, one could randomize the time course of the characterizing measure and keep the original seizure times fixed. For this purpose, the technique of constrained randomization [9] could readily be employed.

Further studies are underway which apply seizure time surrogates in combination with different seizure prediction statistics and to larger samples of the EEG data to further elucidate the problem of preictal state detection [17]. In this context, we expect seizure time surrogates to be a powerful tool to differentiate statistics unsuited for a detection of the preictal state from more promising approaches.

We are grateful to Peter Grassberger for carefully reading this manuscript and for valuable discussions. C.E.E., T.K., K.L., F.M., and C.R. acknowledge support from the Deutsche Forschungsgemeinschaft.
[1] S. Viglione and G. Walsh, Electroencephalogr. Clin. Neurophysiol. 39, 435 (1975); Z. Rogowski, I. Gath, and E. Bental, Biol. Cybern. 42, 9 (1981); J. Gotman et al., Epilepsia 23, 432 (1982); A. Siegel, C. Grady, and A. Mirsky, ibid. 23, 47 (1982); L.D. Iasemidis et al., Brain Topogr 2, 187 (1990); C. E. Elger and K. Lehnertz, in Epileptic Seizures and Syndromes, edited by P. Wolf (Libbey and Co, London, 1994), pp. 547552.

[2] K. Lehnertz and C.E. Elger, Phys. Rev. Lett. 80, 5019 (1998).

[3] J. Martinerie et al., Nat. Med. 4, 1173 (1998); I. Osorio, M.G. Frei, and S.B. Wilkinson, Epilepsia 39, 615 (1998); F. Mormann et al., Physica D 144, 358 (2000); A. Petrosian et al., Neurocomputing 30, 201 (2000); K.K. Jerger et al., J. Clin. Neurophysiol. 18, 259 (2001); L.D. Iasemidis et al., J. Combinatorial Optimization 5, 9 (2001); M. Le Van Quyen et al., Lancet 357, 183 (2001); K. Schindler et al., Clin. Neurophysiol. 113, 604 (2002); V. Navarro et al., Brain 125, 640 (2002); B. Litt et al., Neuron 30, 183 (2001).

[4] R.G. Andrzejak et al., Epilepsia 41, Suppl. 7202 (2000).

[5] H. Kantz and T. Schreiber, Nonlinear Time Series Analysis
(Cambridge University Press, Cambridge, U.K., 1997).

[6] A.R. Osborne and A. Provenzale, Physica D 35, 357 (1989).

[7] J. Theiler et al., Physica D 58, 77 (1992).

[8] J. Theiler, Phys. Lett. A 196, 335 (1995).

[9] T. Schreiber, Phys. Rev. Lett. 80, 2105 (1998).

[10] P. Rapp et al., Phys. Lett. A 192, 27 (1994); J. Timmer, Phys. Rev. E 58, 5153 (1998); D. Kugiumtzis, ibid. 60, 2808 (1999); P. Rapp et al., Int. J. Bifurcation Chaos Appl. Sci. Eng. 11, 983 (2001).

[11] Y.Y. Kagan, Geophys. J. Int. 131, 505 (1997).

[12] Surgical Treatment of the Epilepsies, edited by J. Engel, Jr. (Raven Press, New York, 1993).

[13] R.G. Andrzejak et al., Epilepsy Res. 44, 129 (2001).

[14] D.T. Kaplan and L. Glass, Phys. Rev. Lett. 68, 427 (1992).

[15] T. Schreiber and A. Schmitz, Phys. Rev. Lett. 77, 635 (1996).

[16] F. Takens, in Dynamical Systems and Turbulence, edited by D. A. Rand and L.-S. Young (Springer-Verlag, Berlin, 1980), Lecture Notes in Mathematics, Vol. 898, pp. 366-381.

[17] F. Mormann et al., Phys. Rev. E (to be published). 